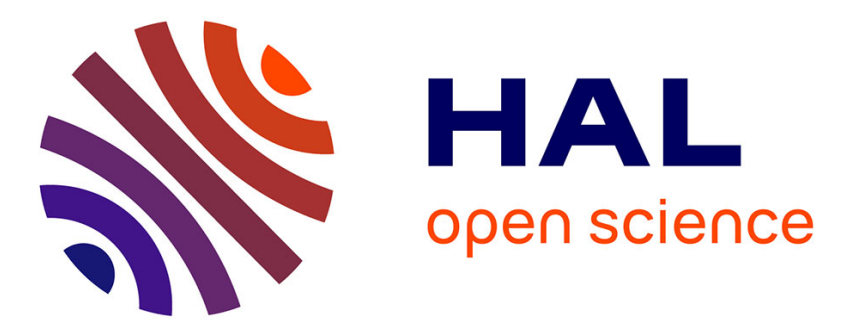

\title{
Asymmetry relations and effective temperatures for biased Brownian gyrators
}

Sara Cerasoli, Victor Dotsenko, Gleb Oshanin, Lamberto Rondoni

\section{To cite this version:}

Sara Cerasoli, Victor Dotsenko, Gleb Oshanin, Lamberto Rondoni. Asymmetry relations and effective temperatures for biased Brownian gyrators. Physical Review E , 2018, 98 (4), 10.1103/PhysRevE.98.042149 . hal-02323097

\section{HAL Id: hal-02323097 https://hal.science/hal-02323097}

Submitted on 21 Oct 2019

HAL is a multi-disciplinary open access archive for the deposit and dissemination of scientific research documents, whether they are published or not. The documents may come from teaching and research institutions in France or abroad, or from public or private research centers.
L'archive ouverte pluridisciplinaire $\mathbf{H A L}$, est destinée au dépôt et à la diffusion de documents scientifiques de niveau recherche, publiés ou non, émanant des établissements d'enseignement et de recherche français ou étrangers, des laboratoires publics ou privés. 


\title{
Asymmetry relations and effective temperatures for biased Brownian gyrators
}

\author{
Sara Cerasoli, ${ }^{1}$ Victor Dotsenko, ${ }^{2,3}$ Gleb Oshanin, ${ }^{2, *}$ and Lamberto Rondoni ${ }^{4,5,6, \dagger}$ \\ ${ }^{1}$ Dipartimento di Fisica, Università degli Studi di Torino, via P. Giuria 1, 10125 Torino, Italy \\ ${ }^{2}$ Sorbonne Université, CNRS, Laboratoire de Physique Théorique de la Matière Condensée, UMR CNRS 7600, 75252 Paris Cedex 05, France \\ ${ }^{3}$ L.D. Landau Institute for Theoretical Physics, 119334 Moscow, Russia \\ ${ }^{4}$ Dipartimento di Scienze Matematiche, Dipartimento di Eccellenza 2018-2022, Politecnico di Torino, \\ Corso Duca degli Abruzzi 24, 10129 Torino, Italy \\ ${ }^{5}$ INFN, Sezione di Torino, Via P. Giuria 1, 10125 Torino, Italy \\ ${ }^{6}$ Malaysia-Italy Centre of Excellence for Mathematical Sciences, Universiti Putra Malaysia, 43400 Seri Kembangan, Selangor, Malaysia
}

(Received 6 August 2018; published 31 October 2018)

\begin{abstract}
We focus on a paradigmatic two-dimensional model of a nanoscale heat engine, the so-called Brownian gyrator, whose stochastic dynamics is described by a pair of coupled Langevin equations with different temperature noise terms. This model is known to produce a curl-carrying non-equilibrium steady-state with persistent angular rotations. We generalize the original model introducing constant forces doing work on the gyrator, for which we derive exact asymmetry relations, that are reminiscent of the standard fluctuation relations. Unlike the latter, our relations concern instantaneous and not time averaged values of the observables of interest. We investigate the full two-dimensional dynamics as well as the dynamics projected on the $x$ and $y$ axes, so that information about the state of the system can be obtained from just a part of its degrees of freedom. Such a state is characterized by effective "temperatures" that can be measured in nanoscale devices, but do not have a thermodynamic nature. Remarkably, the effective temperatures appearing in full dynamics are distinctly different from the ones emerging in its projections, confirming that they are not thermodynamic quantities, although they precisely characterize the state of the system.
\end{abstract}

DOI: 10.1103/PhysRevE.98.042149

\section{INTRODUCTION}

While in the past statistical physics has been mainly devoted to the microscopic basis of the macroscopic behavior, present day research is largely addressing "small" or nonthermodynamic systems, which either evolve spontaneously or are subjected to external drivings and constraints. Unlike macroscopic systems, that are by and large described by thermodynamics and linear response theory, these systems are still hard to be framed within a comprehensive theory.

In fact, macroscopic observations amount to drastic projections from highly dimensional spaces to spaces of observables that consist of just a few dimensions. This is the reason why thermodynamics is so universal; those projections lose an enormous amount of information about the microscopic dynamics, hence the properties of observables only minimally depend on such microscopic details, provided a few conditions are met. Basically, it suffices that atomic forces are short ranged and repulsive. Then, universality as well as the equivalence of ensembles are established, and the resulting theory of macroscopic objects very generally holds. On the contrary, the behavior of systems made of a nonthermodynamic number of elementary constituents strongly

\footnotetext{
${ }^{*}$ Corresponding author: oshanin@1ptmc.jussieu.fr

${ }^{\dagger}$ Present address: Civil and Environmental Engineering Department and Princeton Environmental Institute, Princeton University, Princeton, NJ 08540, USA.
}

depends on all defining parameters, and a theory as widely applicable as thermodynamics can hardly be envisaged.

Nevertheless, one common facet of such systems is that their observables undergo non-negligible fluctuations. Therefore, the seminal paper [1] represents a pioneering attempt towards a unified theory of fluctuating phenomena [2,3]. Its chief result is called fluctuation relation (FR), and it constitutes one of the first exact results obtained for systems which are almost arbitrarily far from equilibrium. Close to equilibrium the FR reproduces the Green-Kubo and Onsager relations $[4,5]$. Also, transient relations provide a method to investigate equilibrium properties of given systems, by means of non-equilibrium experiments, closing the circle with the fluctuation dissipation relation, that yields non-equilibrium properties by means of equilibrium experiments. Various derivations have been given for such an asymmetry relation, that is informally written as

$$
\frac{\operatorname{Prob}\left(\sigma_{\tau} \approx A\right)}{\operatorname{Prob}\left(\sigma_{\tau} \approx-A\right)} \approx e^{\beta_{\mathrm{eff}} A}, \quad \sigma_{\tau}=\int_{0}^{\tau} \mathbf{J} \cdot \mathbf{F} \mathrm{d} t,
$$

where $\sigma_{\tau}$ is the power dissipated in a long time interval $\tau$, for a system driven by a force $\mathbf{F}$ that produces the fluctuating current $\mathbf{J}$, and $\operatorname{Prob}\left(\sigma_{\tau} \approx A\right)$ is the probability that $\sigma_{\tau}$ is close to $A$. For homogeneous systems at temperature $T$, measured in units of the Boltzmann constant $k_{B}, \beta_{\text {eff }}$ equals $1 / T$ but in general it contains an "effective temperature" [6-8] that depends on the case at hand.

Given the importance and success of such a FR [9-12], a wide variety of analogous results has been derived in quite 
different contexts. In particular, we now have FRs for dynamical systems and stochastic processes, for classical and quantum systems, for transient, steady states and ageing systems, for global and local quantities, for steady and time dependent drivings. Such FRs concern a variety of observables, the most common involving work, heat, and energy dissipation. In particular, steady state FRs for thermodynamic quantities need $\tau$ large compared to the microscopic dynamics time scales. Although fluctuations are not normally observable in macroscopic systems, FRs have been verified in gravitational wave detectors, that are indeed meant to reveal microscopic fluctuations in macroscopic systems [22]. The literature on FRs is abundant [11-21].

Despite so much activity, it still remains to clarify the extent to which symmetries analogous to Eq. (1) hold for nonthermodynamics or even non-physical phenomena, such as population dynamics, when described by models analogous to the physical ones. However, the thermodynamic interpretation will not apply, in general.

In this respect, exactly solvable models are most useful, because they provide meaningful benchmarks for a more general analysis, unveiling at the same time the complexity and non-trivial aspects of the problem. To this end, we focus on the exactly solvable model of a Brownian gyrator widely studied in the past (see below), due its non-trivial non-equilibrium steady-state (NESS) with a non-zero curl. We generalize this model here introducing constant forces that do work on the gyrator. This does not merely add another variable to the parameter space, but allows us to go well beyond the previous analyses and probe asymmetry relations, reminiscent of the standard FRs, for such a curl-carrying NESS. We derive exact asymmetry relations, characterizing the state of the system, for the two-dimensional as well as for the one-dimensional projected dynamics, revealing a peculiar behavior for the corresponding effective "temperatures". The functional form of the latter appears perhaps to be even more striking than the one obtained for the exchanged heat in transient and stationary regimes [8]. Moreover, our relations are more detailed than standard ones, because they concern instantaneous values, rather than averaged values of the observables of interest.

\section{MODEL}

Stochastic dynamics of a biased Brownian gyrator is described by two coupled Langevin equations:

$$
\begin{aligned}
& \dot{x}=F_{x}-\frac{\partial U_{0}}{\partial x}(x, y)+\zeta_{x}(t), \\
& \dot{y}=F_{y}-\frac{\partial U_{0}}{\partial y}(x, y)+\zeta_{y}(t),
\end{aligned}
$$

in which the viscosity $\eta=1, \mathbf{F}=\left(F_{x}, F_{y}\right)$ is a constant force exerting a regular bias on the gyrator (both $F_{x}$ and $F_{y}$ can independently take any real value), and the potential $U_{0}(x, y)$ has a generic parabolic form

$$
U_{0}(x, y)=\frac{x^{2}}{2}+\frac{y^{2}}{2}+u x y,
$$

where the coupling constant $u$ obeys $u^{2}<1$, for reasons to be clarified below. Lastly, $\zeta_{\alpha}(t),(\alpha=x, y)$, are Gaussian white noises with zero mean and covariances functions:

$$
\overline{\zeta_{\alpha}(t) \zeta_{\beta}\left(t^{\prime}\right)}=2 T_{\alpha} \delta_{\alpha, \beta} \delta\left(t-t^{\prime}\right)
$$

with $\delta_{\alpha, \beta}=0$ for $\alpha \neq \beta$, and $\delta_{\alpha, \beta}=1$ for $\alpha=\beta$. In general, $T_{x} \neq T_{y}$. Note as well that the minimum $O_{m}=\left(x_{m}, y_{m}\right)$ of the effective potential $U(x, y)=-F_{x} x-F_{y} y+U_{0}(x, y)$, in which the Langevin dynamics takes place, is located at

$$
x_{m}=\frac{F_{x}-u F_{y}}{1-u^{2}}, \quad y_{m}=\frac{F_{y}-u F_{x}}{1-u^{2}},
$$

i.e., it does not lie in the origin $(0,0)$, unless $F_{x}=0$ and $F_{y}=$ 0 . For $u \rightarrow 1^{-}$, both $\left|x_{m}\right|$ and $\left|y_{m}\right|$ tend to infinity.

Before we proceed, we note that the system described by Eq. (2) can be viewed from a different perspective, for which the non-thermodynamic character of the effective temperatures derived below is most apparent. Namely, the Langevin equations (2) can be deemed to describe the temporal evolution of, say, the specific densities $x$ and $y$ of two interacting populations which are continuously introduced into the system by two independent random sources with mean intensities $F_{x}$ and $F_{y}$ and fluctuations having different amplitudes- $T_{x}$ and $T_{y}$. The species of the populations have their intrinsic equal life-times [terms $-x$ and $-y$ in the right-hand side of Eq. (2)] and share some common resources, such that, for $0<u<1$, an increase of the $x$ - or $y$-population prompts a decrease of the $y$ - or $x$-population [terms $-u y$ and $-u x$ in the right-hand side of Eq. (2)]. As we set out to show in what follows, dynamics of such coupled populations in this seemingly simple model appears to be very non-trivial.

Reference [23], which addressed the notion of effective temperatures [24], first noted that the unbiased case, $F_{x}=F_{y}=0$, can be solved exactly in the $t \rightarrow \infty$ limit. In Ref. [28], the model was seen as the simplest nanoscale heat engine; its average torque was determined analytically, and experimental realizations were discussed, including devices with (a) an anisotropic black-body radiation; (b) an electrical heat bath made of two resistors at different temperatures; (c) two heat baths-a usual fluid environment with isotropic properties and an unusual one emitting thermal fluctuations in a preferential direction. In Refs. [29,30], an experimental scenario close to (b) was realized, and the exchanged heat and work were measured. The theoretical analysis of Refs. [29,30], consistent with the experimental evidence, was based on a variation of Eqs. (2), (4), and (3) suitable for the evolution of voltages in coupled resistors, produced fluctuation relations for the unbiased case. In Ref. [8] this analysis was extended to obtain both transient and steady states fluctuation relations, that respectively hold for all and only for asymptotic observation time intervals, in accordance with our discussion above. An experimental realization of gyrators was developed also in Ref. [31], in a framework that can be generalized to include our model settings, that being, constant bias exerted on the gyrator.

Reference [32] used Eqs. (2), (4), and (3) with $F_{x}=F_{y}=$ 0 to investigate the relevance of information contained in cross correlations among different degrees of freedom in nonequilibrium systems. In turn, Ref. [33] and later Ref. [34] focused on non-equilibrium currents and provided explicit expressions for their curl, for the mean angular velocity of the rotational motion [33,34], and for the variance of the 
latter [34]. Reference [34] also argued that Eqs. (2), (4), and (3) may describe a rotation of clouds of cold atoms, following laser detuning imbalance during the cooling phase, which leads to different temperatures along the different cooling axis. Lastly, Refs. [33,34] numerically analyzed the time-averaged angular velocity $\omega$ for a single long trajectory $\rho_{t}$, demonstrating that $\omega$ converges to its ensemble-averaged counterpart in the limit of a long observation time.

Our model with constant forces doing work on the gyrator generalizes the previous ones, so that more experimental devices, including, e.g., nanomechanical resonators [25,26], and even population dynamics, can be considered [35]. Like other investigations of fluctuation relations led to results of more general interest (e.g., novel response theories $[19,27]$ ), our asymptotic asymmetry relations produce a new form of effective temperature, expected to be measurable, e.g., in settings similar to those of Refs. [26,29,30].

\section{RESULTS}

In the NESS, the Fokker-Planck equation for the probability density function $P(x, y)$ takes the form

$$
\operatorname{div}(\mathbf{j})=\frac{\partial j_{x}}{\partial x}+\frac{\partial j_{y}}{\partial y}=0
$$

where the current $\mathbf{j}=\left(j_{x}, j_{y}\right)$ is defined by

$$
\begin{aligned}
& j_{x}=T_{x} \frac{\partial P(x, y)}{\partial x}+P(x, y) \frac{\partial U(x, y)}{\partial x}, \\
& j_{y}=T_{y} \frac{\partial P(x, y)}{\partial x}+P(x, y) \frac{\partial U(x, y)}{\partial x} .
\end{aligned}
$$

The analytical solution of Eqs. (6) and (7) reads

$$
\begin{gathered}
P(x, y)=Z^{-1} \exp \left(-\frac{2}{4 T_{x} T_{y}+u^{2} \Delta_{T}^{2}}\left(\left(T_{y}+\frac{u^{2} \Delta_{T}}{2}\right) x^{2}+\left(T_{x}-\frac{u^{2} \Delta_{T}}{2}\right) y^{2}\right.\right. \\
\left.\left.+u\left(T_{x}+T_{y}\right) x y-\left(2 F_{x} T_{y}+u F_{y} \Delta_{T}\right) x-\left(2 F_{y} T_{x}-u F_{x} \Delta_{T}\right) y\right)\right), \\
Z=\pi \sqrt{\frac{4 T_{x} T_{y}+u^{2} \Delta_{T}^{2}}{1-u^{2}}} \exp \left(\frac{2\left(F_{y}^{2} T_{x}+F_{x}^{2} T_{y}\right)-2 u F_{x} F_{y}\left(T_{x}+T_{y}\right)+u^{2}\left(F_{x}^{2}-F_{y}^{2}\right) \Delta_{T}}{\left(1-u^{2}\right)\left(4 T_{x} T_{y}+u^{2} \Delta_{T}^{2}\right)}\right)
\end{gathered}
$$

with $\Delta_{T}=T_{x}-T_{y}$. For $T_{x}=T_{y}=T$, one has the required Boltzmann form $P(x, y)=Z_{0}^{-1} \exp (-U(x, y) / T)$, while for $T_{x} \neq T_{y}$ and $F_{x}=F_{y}=0$ the result reported in $[23,28$ $30,32-34]$ is recovered. Equation (9) shows that the normalization $Z$ exists only for $u^{2}<1$, as noted above.

In Figs. 1(a) and 1(d), we present the bivariate pdf $P(x, y)$ for $u=3 / 4$, and for two different choices of temperatures and forces. We observe that $P(x, y)$ has a maximum at $O_{m}$ and is strongly elongated along its principal axis, being considerably shorter in the perpendicular direction. The variances of the distribution relative to $O_{m}$ are given explicitly for all values of $T_{x}$ and $T_{y}$ by

$$
\begin{aligned}
& \sigma_{x}^{2}=\left\langle\left(x-x_{0}\right)^{2}\right\rangle=\frac{1}{1-u^{2}}\left(T_{x}-\frac{u^{2}}{2} \Delta_{T}\right), \\
& \sigma_{y}^{2}=\left\langle\left(y-y_{0}\right)^{2}\right\rangle=\frac{1}{1-u^{2}}\left(T_{y}+\frac{u^{2}}{2} \Delta_{T}\right),
\end{aligned}
$$

where the angular brackets, here and henceforth, denote averages with respect to the distribution of Eqs. (8) and (9). Note: $\sigma_{x}^{2}$ and $\sigma_{y}^{2}$ are independent of $F_{x}$ and $F_{y}$, and they have opposite trends under variations of $\Delta_{T}$ : for $\Delta_{T}>0\left(\Delta_{T}<0\right)$ $\sigma_{x}^{2}$ decreases (increases), while $\sigma_{y}^{2}$ increases (decreases), for growing $\left|\Delta_{T}\right|$. When $u \rightarrow 1^{-}$with $T_{x}$ and $T_{y}$ fixed, both variances grow without bounds.

In Figs. 1(b) and 1(e) the vector plot of $\mathbf{j}(x, y)$ is given, showing that its circulation around $O_{m}$, along the closed orbits defined by $P(x, y)=$ const. The direction of such a circulation is determined by the sign of $\Delta_{T}$ only.

To further characterize $\mathbf{j}$, we compute its curl:

$$
\operatorname{curl}(\mathbf{j})=\frac{\partial j_{y}}{\partial x}-\frac{\partial j_{x}}{\partial y}=\Delta_{T} u A(x, y) P(x, y),
$$

where $A(x, y)$ is a quadratic form of $x$ and $y$, with coefficients depending in a complicated fashion on the system parameters, which we omit here. Clearly, $\operatorname{curl}(\mathbf{j})$ is rather non-trivial, as observed in Figs. 1(c) and 1(f). In particular, it qualitatively changes when $T_{x}$ and $T_{y}$ are changed: for $T_{x}=20$ and $T_{y}=1$ it has a hump and two deeps; while for $T_{x}=0$ and $T_{y}=5$ the hump becomes a deep, and the deeps turn into humps. This can be seen from the following expression for $\operatorname{curl}(\mathbf{j})$ at $O_{m}$ :

$$
\left.\operatorname{curl}(\mathbf{j})\right|_{x=x_{m}, y=y_{m}}=\frac{2 \sqrt{1-u^{2}}\left(T_{x}+T_{y}\right)}{\pi \sqrt{4 T_{x} T_{y}+u^{2} \Delta_{T}^{2}}} u \Delta_{T} .
$$

Here, $u \Delta_{T}>0$ implies a maximum of the curl at the minimum of $U$, while if the inverse inequality holds, the minimum of $U$ implies minimal curl. Note that Eq. (13) does not depend on the values of $F_{x}$ and $F_{y}$.

The ensemble-average angular velocity $\langle\omega\rangle$ is given by $\langle\omega\rangle=-\pi u \Delta_{T} / Z$, with $Z$ defined by Eq. (9). In contrast to the variances, Eqs. (10), the curl and the rotation velocity strongly depend on $\mathbf{F}$. In particular, $Z$ brings an exponential dependence on $F_{x}$ and $F_{y}$ to $\omega$, which vanishes very rapidly when any of the two forces increases. Indeed, the length of orbits increases with an increase of force, which also means a longer time to go round. 


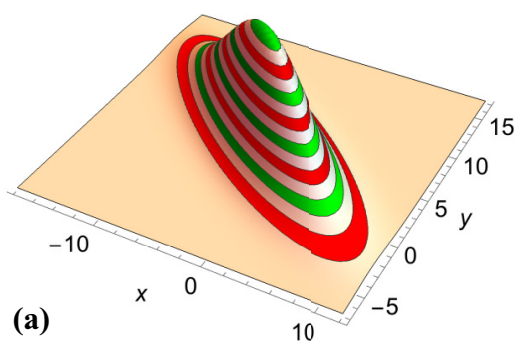

(b)
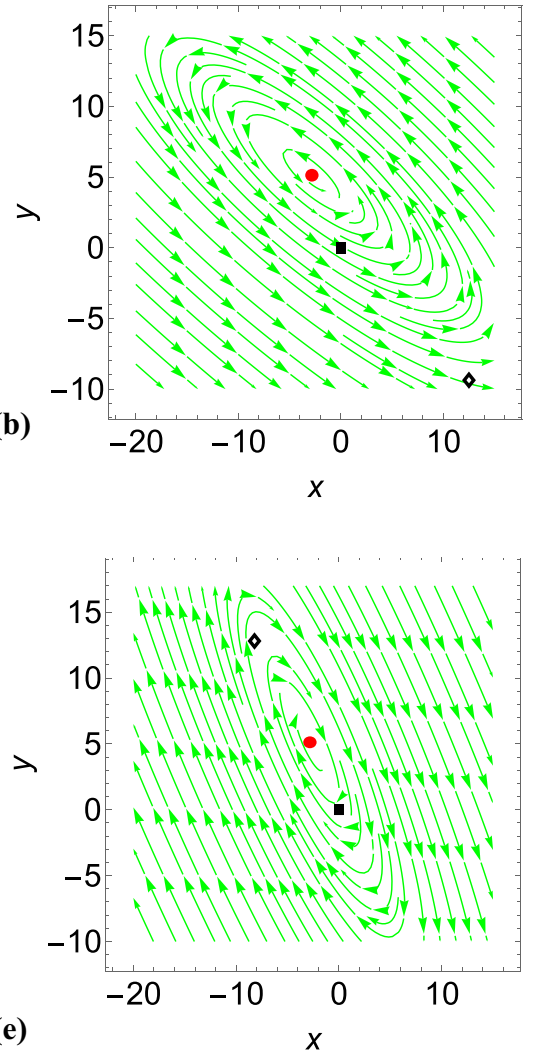

(d)

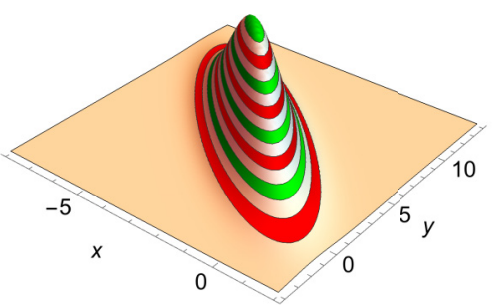

(e)
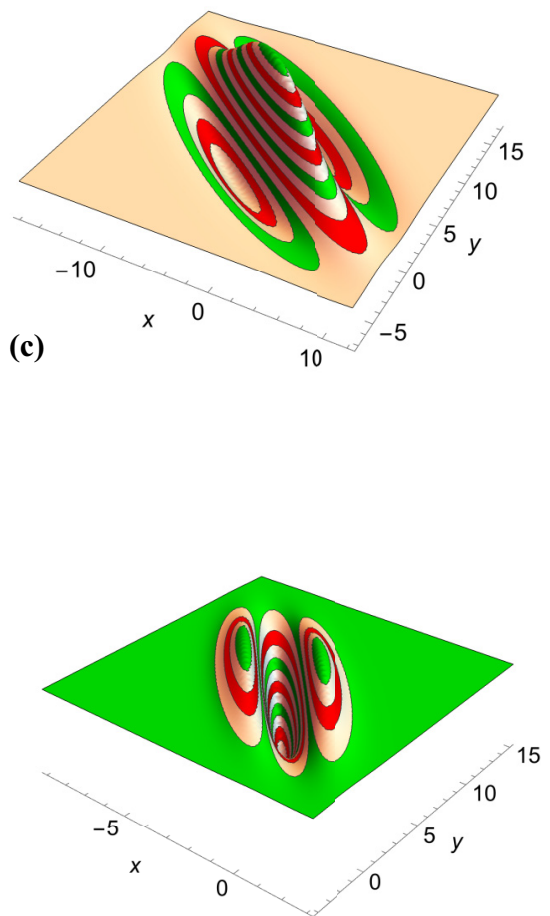

(f)

FIG. 1. The joint pdf $P(x, y)$ (a) and (d), Eq. (8), the current $\mathbf{j}$ (b) and (e), Eqs. (7), and the curl of the current $\mathbf{j}$ (c) and (f), Eq. (12), as functions of $x$ and $y$ for $u=3 / 4$ and for different choices of $T_{x}, T_{y}, F_{x}$, and $F_{y}$. In the first row, $T_{x}=20, T_{y}=1, F_{x}=1$, and $F_{y}=3$. In the second row, $T_{x}=0, T_{y}=5, F_{x}=1$, and $F_{y}=3$. In (b) and (e), the full square denotes the origin, the filled circle (red) denotes the minimum $O_{m}$ of the potential, Eqs. (5), while the diamond shows the location of point of isometry $O_{i s}$ with coordinates $x_{i s}=u \Delta_{T}\left(2 F_{y} T_{x}+\right.$ $\left.F_{x}\left(T_{x}+T_{y}\right)-F_{y} u^{2} \Delta_{T}\right) /\left(1-u^{2}\right)\left(4 T_{x} T_{y}+u^{2} \Delta_{T}^{2}\right)$ and $y_{i s}=-u \Delta_{T}\left(2 F_{x} T_{y}+F_{y}\left(T_{x}+T_{y}\right)+F_{x} u^{2} \Delta_{T}\right) /\left(1-u^{2}\right)\left(4 T_{x} T_{y}+u^{2} \Delta_{T}^{2}\right)[\mathrm{see}$ the text above Eq. (17)].

Now, the pdf $P(x, y)$ obeys the asymmetry relation

$$
\begin{aligned}
& \ln \frac{P(x, y)}{P(-x,-y)} \\
& \quad=\frac{2 F_{x} x}{T_{\mathrm{eff}}^{(x)}}+\frac{2 F_{y} y}{T_{\mathrm{eff}}^{(y)}}+u\left(\frac{1}{T_{\mathrm{eff}}^{(y)}}-\frac{1}{T_{\mathrm{eff}}^{(x)}}\right)\left(F_{y} x-F_{x} y\right) .
\end{aligned}
$$

Note that $F_{x} x$ and $F_{y} y$ are the works done by $\mathbf{F}$ along the directions $x$ ad $y$, for points starting at $(0,0)$, while $T_{\text {eff }}^{(x)}$ and $T_{\text {eff }}^{(y)}$ are effective temperatures, given explicitly by

$$
\begin{aligned}
& T_{\mathrm{eff}}^{(x)}=T_{x}+\frac{u^{2}}{4} \frac{\left(T_{x}-T_{y}\right)^{2}}{T_{y}}, \\
& T_{\mathrm{eff}}^{(y)}=T_{y}+\frac{u^{2}}{4} \frac{\left(T_{x}-T_{y}\right)^{2}}{T_{x}} .
\end{aligned}
$$

For reservoirs with $T_{x}=T_{y}=T$, both $T_{\mathrm{eff}}^{(x)}$ and $T_{\mathrm{eff}}^{(y)}$ equal $T$, while for $T_{x} \neq T_{y}$, the effective temperatures are both larger than the thermodynamic temperatures of their respective reservoirs. Remarkably, a similar effect was experimentally observed and theoretically interpreted in Ref. [36]. Furthermore, letting the reservoir temperature $T_{x}$ vanish, so that the dynamics of $x$ is totally subordinated to that of $y$, we observe that $T_{\text {eff }}^{(x)}$ tends to $T_{\text {eff }}^{(x)}=u^{2} T_{y} / 4$, while the effective temperature $T_{\mathrm{eff}}^{(y)}$ diverges. In contrast, the variances in Eqs. (10) stay finite when $T_{x} \rightarrow 0$. Note, as well, that the divergence of $T_{\text {eff }}^{(y)}$ derives from taking the $t \rightarrow \infty$ limit before the $T_{x} \rightarrow 0$ limit. At finite times, the expression (14) should contain finite effective temperatures, with presumably exponentially fast divergence along $y$ with time. Finally, we can write

$$
\frac{T_{\text {eff }}^{(x)}+T_{\mathrm{eff}}^{(y)}}{2}=\left(1+\frac{u^{2}}{4} \frac{\left(T_{x}-T_{y}\right)^{2}}{T_{x} T_{y}}\right) \frac{T_{x}+T_{y}}{2},
$$

showing that the mean effective temperature is always greater than the mean temperature and, that it becomes infinitely large when either $T_{x}$ or $T_{y}$ vanish.

Equation (14) refers to the origin of the $(x, y)$-plane, but an analogous property can be obtained for any other point. Referring to $O_{m}$, one has $\ln \left(P\left(x=x_{m}+\delta_{x}, y=y_{m}+\delta_{y}\right) / P(x=\right.$ $\left.\left.x_{m}-\delta_{x}, y=y_{m}-\delta_{y}\right)\right) \equiv 0$, for arbitrary $\delta_{x}$ and $\delta_{y}$. Also, there is a single point, $O_{i s}=\left(x_{i s}, y_{i s}\right)$, such that Eq. (14) takes the isometric form

$$
\ln \frac{P\left(x=x_{i s}+\delta_{x}, y=y_{i s}+\delta_{y}\right)}{P\left(x=x_{i s}-\delta_{x}, y=y_{i s}-\delta_{y}\right)}=\frac{2 F_{x} \delta_{x}}{T_{\mathrm{eff}}^{(x)}}+\frac{2 F_{y} \delta_{y}}{T_{\mathrm{eff}}^{(y)}},
$$

for arbitrary $\delta_{x}$ and $\delta_{y}$, see Figs. 1(b) and 1(e). 
Marginalizing $P(x, y)$,

$$
P(x)=\int_{-\infty}^{\infty} P(x, y) d y, \quad P(y)=\int_{-\infty}^{\infty} P(x, y) d x,
$$

one obtains the following pair of asymmetry relations:

$$
\begin{aligned}
& \ln \frac{P(x)}{P(-x)}=\frac{2 x F_{x}}{\tau_{\mathrm{eff}}^{(x)}}-2 u \frac{F_{y} x}{\tau_{\mathrm{eff}}^{(x)}}, \\
& \ln \frac{P(y)}{P(-y)}=\frac{2 y F_{y}}{\tau_{\mathrm{eff}}^{(y)}}-2 u \frac{F_{x} y}{\tau_{\mathrm{eff}}^{(y)}}
\end{aligned}
$$

with effective temperatures $\tau_{\text {eff }}^{(x)}$ and $\tau_{\text {eff }}^{(y)}$ given by

$$
\begin{aligned}
& \tau_{\mathrm{eff}}^{(x)}=T_{x}-\frac{u^{2}}{2}\left(T_{x}-T_{y}\right), \\
& \tau_{\mathrm{eff}}^{(y)}=T_{y}+\frac{u^{2}}{2}\left(T_{x}-T_{y}\right) .
\end{aligned}
$$

Unlike $T_{\text {eff }}^{(x)}$ and $T_{\text {eff }}^{(y)}, \tau_{\text {eff }}^{(x)}$ and $\tau_{\text {eff }}^{(y)}$ (a) can be smaller than $T_{x}$ and $T_{y}$; (b) do not diverge if either $T_{x}$ or $T_{y}$ vanishes; (c) are equal, up to a scale factor $1-u^{2}$, to the variances of the pdf $P(x, y)$, Eq. (10), and (d) they obey

$$
\frac{\tau_{\mathrm{eff}}^{(x)}+\tau_{\mathrm{eff}}^{(y)}}{2} \equiv \frac{T_{x}+T_{y}}{2},
$$

i.e., their mean is identically equal to the mean reservoirs' temperature. Interestingly enough, the effective temperatures emerging in the projected dynamics exhibit a completely different and somewhat trivial behavior as compared to the one appearing in the full dynamics. This observation illustrates the statement made in the beginning of our work that projecting the dynamics from higher dimensional spaces onto the individual components and marginalizing the distributions lead to a significant loss of information.

\section{CONCLUSIONS}

To conclude, we studied a model of a Brownian gyrator subject to work-doing constant forces. We presented an exact solution of this model in the steady-state and showed that the probability density function obeys an asymmetry relation that contains effective temperatures, one of which may become arbitrarily large, mimicking certain experimental observations [36]. The effective temperatures of the projected dynamics, on the contrary, cannot exceed the sum of the reservoirs temperatures. Our results provide a novel important insight on the notion and behavior of effective temperatures in outof-equilibrium conditions. In our case, they can be measured thanks to our relations, when the PDFs of the coordinates are known from, e.g., experimental measurements, such as those performed in Ref. [31]. Lastly, we stress that the quantities discussed above may be interpreted physically, as thermodynamic quantities, in particular. However, this interpretation is not necessary, especially when small systems are observed over short times. Furthermore, relations for time-averaged quantities can be obtained by time integration, from our more detailed instantaneous relations.

\section{ACKNOWLEDGMENTS}

S.C. wishes to thank LPTMC, Sorbonne Université, for a warm hospitality during her Master Degree training stage in July 2018 when this work had been performed, and also acknowledges support from Università degli Studi di Torino. L.R. acknowledges partial support by Ministero dell'Istruzione, dell'Università e della Ricerca (MIUR, Italy) grant Dipartimenti di Eccellenza 2018-2022.
[1] D. J. Evans, E. G. D. Cohen, and G. P. Morriss, Probability of Second Law Violations in Shearing Steady States, Phys. Rev. Lett. 71, 2401 (1993).

[2] G. Gallavotti, Ergodicity, ensembles, irreversibility in Boltzmann and beyond, J. Stat. Phys. 78, 1571 (1995).

[3] G. Gallavotti and E. G. D. Cohen, Dynamical ensembles in stationary states, J. Stat. Phys. 80, 931 (1995).

[4] G. Gallavotti, Extension of Onsager's Reciprocity to Large Fields and the Chaotic Hypothesis, Phys. Rev. Lett. 77, 4334 (1996).

[5] D. J. Evans, D. J. Searles, and L. Rondoni, Application of the Gallavotti-Cohen fluctuation relation to thermostated steady states near equilibrium, Phys. Rev. E 71, 056120 (2005).

[6] L. Cugliandolo, The effective temperature, J. Phys. A: Math. Theor. 44, 483001 (2011).

[7] A. Puglisi, A. Sarracino, and A. Vulpiani, Temperature in and out of equilibrium: A review of concepts, tools and attempts, Phys. Rep. 709-710, 1 (2017).

[8] A. Bérut, A. Imparato, A. Petrosyan, and S. Ciliberto, Theoretical description of effective heat transfer between two viscously coupled beads, Phys. Rev. E 94, 052148 (2016).
[9] D. J. Searles, D. J. Evans, and L. Rondoni, The steady state fluctuation relation for the dissipation function, J. Stat. Phys. 128, 1337 (2007).

[10] J. R. Gomez-Solano, L. Bellon, A. Petrosyan, and S. Ciliberto, Steady state fluctuation relations for systems driven by an external random force, Europhys. Lett. 89, 60003 (2010).

[11] U. Seifert, Stochastic thermodynamics, fluctuation theorems, and molecular machines, Rep. Prog. Phys. 75, 126001 (2012).

[12] S. Ciliberto, Experiments in Stochastic Thermodynamics: Short History and Perspective, Phys. Rev. X 7, 021051 (2017).

[13] L. Rondoni and C. Mejía-Monasterio, Fluctuations in nonequilibrium statistical mechanics: models, mathematical theory, physical mechanisms, Nonlinearity 20, R1 (2007).

[14] U. M. B. Marconi, A. Puglisi, L. Rondoni, and A. Vulpiani, Fluctuation-dissipation: Response theory in statistical physics, Phys. Rep. 461, 111 (2008).

[15] G. Gallavotti, Nonequilibrium and Irreversibility (Springer, Berlin, 2014).

[16] J. Kurchan, Non-equilibrium work relations, J. Stat. Mech. (2007) P07005. 
[17] V. Jaksić, C.-A. Pillet, and L. Rey-Bellet, Entropic fluctuations in statistical mechanics: I. Classical dynamical systems, Nonlinearity 24, 699 (2011).

[18] D. J. Evans and D. J. Searles, The fluctuation theorem, Adv. Phys. 51, 1529 (2002).

[19] D. J. Evans, S. R. Williams, D. J. Searles, and L. Rondoni, On typicality in nonequilibrium steady states, J. Stat. Phys. 164, 842 (2016).

[20] S. Dal Cengio and L. Rondoni, Broken versus non-broken time reversal symmetry: Irreversibility and response, Symmetry $\mathbf{8}$, 73 (2016).

[21] M. Polettini and M. Esposito, Effective Thermodynamics for a Marginal Observer, Phys. Rev. Lett. 119, 240601 (2017).

[22] M. Bonaldi, L. Conti, P. DeGregorio, L. Rondoni, G. Vedovato, A. Vinante, M. Bignotto, M. Cerdonio, P. Falferi, N. Liguori, S. Longo, R. Mezzena, A. Ortolan, G. A. Prodi, F. Salemi, L. Taffarello, S. Vitale, and J. P. Zendri, Nonequilibrium SteadyState Fluctuations in Actively Cooled Resonators, Phys. Rev. Lett. 103, 010601 (2009).

[23] R. Exartier and L. Peliti, A simple system with two temperatures, Phys. Lett. A 261, 94 (1999).

[24] L. F. Cugliandolo, J. Kurchan, and L. Peliti, Energy flow, partial equilibration, and effective temperatures in systems with slow dynamics, Phys. Rev. E 55, 3898 (1997).

[25] E. Gil-Santos, D. Ramos, A. Jana, M. Calleja, A. Raman, and J. Tamayo, Mass sensing based on deterministic and stochastic responses of elastically coupled nanocantilevers, Nano Lett. 9 , 4122 (2009).

[26] S. Stassi, A. Chiadò, G. Calafiore, G. Palmara, S. Cabrini, and C. Ricciardi, Experimental evidence of Fano resonances in nanomechanical resonators, Sci. Rep. 7, 1065 (2017).

[27] D. Ruelle, General linear response formula in statistical mechanics, and the fluctuation-dissipation theorem far from equilibrium, Phys. Lett. A 245, 17 (1998).

[28] R. Filliger and P. Reimann, Brownian Gyrator: A Minimal Heat Engine on the Nanoscale, Phys. Rev. Lett. 99, 230602 (2007).

[29] S. Ciliberto, A. Imparato, A. Naert, and M. Tanase, Heat Flux and Entropy Produced by Thermal Fluctuations, Phys. Rev. Lett. 110, 180601 (2013).

[30] S. Ciliberto, A. Imparato, A. Naert, and M. Tanase, Statistical properties of the energy exchanged between two heat baths coupled by thermal fluctuations, J. Stat. Mech. (2013) P12014.

[31] A. Argun, J. Soni, L. Dabelow, S. Bo, G. Pesce, R. Eichhorn, and G. Volpe, Experimental realization of a minimal microscopic heat engine, Phys. Rev. E 96, 052106 (2017).

[32] A. Crisanti, A. Puglisi, and D. Villamaina, Nonequilibrium and information: The role of cross correlations, Phys. Rev. E 85, 061127 (2012).

[33] V. Dotsenko, A. Maciolek, O. Vasilyev, and G. Oshanin, Two-temperature Langevin dynamics in a parabolic potential, Phys. Rev. E 87, 062130 (2013).

[34] V. Mancois, B. Marcos, P. Viot, and D. Wilkowski, Twotemperature Brownian dynamics of a particle in a confining potential, Phys. Rev. E 97, 052121 (2018).

[35] S. Cerasoli, V. Dotsenko, G. Oshanin, and L. Rondoni (unpublished).

[36] L. Conti et al., Effects of breaking vibrational energy equipartition on measurements of temperature in macroscopic oscillators subject to heat flux, J. Stat. Mech. (2013) P12003. 